\title{
Improvised Filter Design for Depth Estimation from Single Monocular Images
}

\author{
Aniruddha Das, Vikas Ramnani, Jignesh Bhavsar, and Suman K. Mitra \\ Dhirubhai Ambani Institute of Information and Communication Technology, \\ Gandhinagar, India \\ \{aniruddha_das,vikas_ramnani,jignesh_bhavsar,suman_mitra\}@daiict.ac.in
}

\begin{abstract}
Depth Estimation poses various challenges and has wide range applications. Techniques for depth prediction from static images include binocular vision, focus-defocus, stereo vision and single monocular images unfortunately not much attention has been paid on depth estimation from single image except 1 . We have proposed a method for depth estimation from single monocular images which is based on filters that are used to extract key image features. The filters used have been applied at multiple scales to take into account local and global features. Here an attempt is made to reduce the dimension of feature vector as proposed in 1]. In this paper we have optimized the filters used for texture gradient extraction. This paper also introduces a prediction algorithm whose parameters are learned by repeated correction. The new methodology proposed provides an equivalent quality of result as in [1.
\end{abstract}

\section{Introduction}

Emerging interest in self driven cars, boats and ships [7] has made it necessary for continuous and fast real time depth perception algorithms. Depth Estimation has very wide applications in robotics [3], scene understanding and 3-D reconstruction 9 .

Collaborative Ocular Melanoma Study (COMS) 8] has defined depth perception as the power to discern three-dimensional objects and their relative position in space. Visual perception can be classified into Monocular Vision and Binocular Vision. Binocular Vision uses a pair of images from different angle of the same object to generate depth maps implemented in [6]. This paper deals with depth estimation in monocular vision, that uses a single image.

There are two methods for retrieving depths from monocular digital images. The first method includes using a sequence of closely related stream of images with prior information of the scenes or video for depth estimation. Second method is an algorithm for depth prediction from single independent monocular images. A novel algorithm for depth estimation from images using focus and defocus has been presented by Das and Ahuja [5].

Depth estimation from single monocular images can be implemented using stereo features of images and monocular features of images. Stereo Vision has also been used to generate depth maps from images [10. It has shown excellent

S. Chaudhury et al. (Eds.): PReMI 2009, LNCS 5909, pp. 333-338, 2009.

(C) Springer-Verlag Berlin Heidelberg 2009 
results on the Middlebury stereo evaluation test bed. Saxena et al. [1] presents a discriminately trained MRF for depth estimation from single monocular images. The algorithm presented hereby uses features extracted from single monocular images correlated with depth map to train a machine for depth estimation. The algorithm identifies the key steps involved in estimating depth from discreet and uncorrelated images. Saxena et. al [1] implements a set of 17 filters for computing the feature vector. The feature vector is defined on sub images which are called patches. The feature vector is then used in the formation of a machine learning model to predict depth. They have effectively reduced blocking effect by taking into consideration neighboring patches.

In this paper we have introduced a new learning methodology, after optimizing the feature selection criteria. Section 2 describes feature selection, extraction, learning approach and benefits of new methodology used for depth estimation. Section 3 depicts the comparison of new methodology and Saxena et al. [1].

\section{Proposed Methodology}

Here we are proposing a procedure for depth estimation of outdoor scenes. Note that exactly similar procedure may not be applicable to indoor scenes. The system of depth estimation from single monocular images consists of three basic steps. Initially, a set of images and there corresponding depth maps are gathered. Then a set of suitable features are extracted from the images. Based on the features and the ground truth depth maps a prediction model is learnt. The depth of new images are predicted from the learnt model.

It has been observed that the changes in depths along the row of any image compared to the same along the column is comparatively less. Along the row the depth of the scene observed by camera eye would depend on the angle of camera. On other hand the depth along the column till infinity since the outdoor scene is unbounded due to the presence of sky. Moreover, depths are estimated for small patches within the image. Hence, the feature selection has been done for each patch of the image. On the other hand learning of depth map has been done for a row of patches instead of a single patch. Note that similar argument has been used in [1. Next, the feature selection and learning methodology are discussed. A set of filters are designed to extract features. In particular these filters are expected to extract texture energy, texture gradient and haze. These cues are used because texture energy of objects varies according to distances from the viewers, texture gradient captures the distribution of the direction of edges and haze is used to detect atmospheric light scattering [1]. These three cues help to indicate depths.

Figure 1 indicates a total of 16 such filters used by Saxena et al. 11. The filters used in 1 for texture gradient are mainly edge detection filters. The edge detection filters are primarily directional oriented high pass filters. In this paper, a single Omni- directional high pass filter, in place, of the six directional texture gradient filters [1] is used to compute the texture gradient information. The use of an Omni- directional high pass filter reduces the net size of the feature vector. 


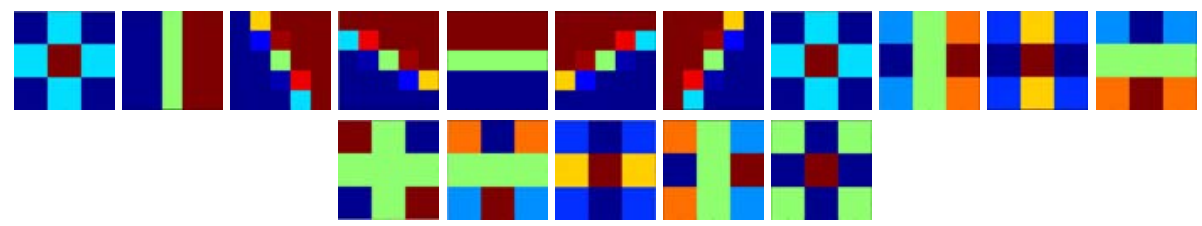

Fig. 1. A set of 16 filters as implemented by Saxena et al. 1]. (The first low pass filter is used twice on two different color channels results in a total of 17 features.)
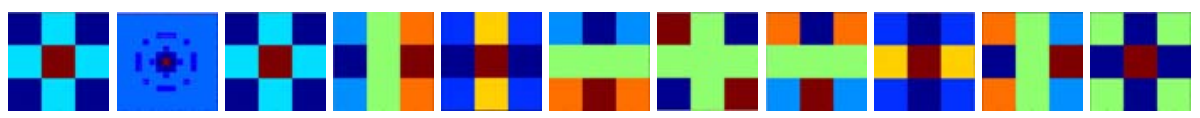

Fig. 2. The set of new filter used for the current experiment. Filter 2 is the new omnidirectional high pass filter changed. (The first low pass filter is used twice on two different color channels which gives a total of 12 features.)

The Omni-directional high pass filter has been shown in filter 2 of Figure 2 . Other filters used are same as in 1 .

In the prior work of Saxena et al. 1] the prediction model assumed to be linear with error distributed as Gaussian and Laplacian. However, no justification of the same has been reasoned out. In our experimentation it has been observed that the relation between the ground truth depth and its prediction could be non linear and hence we are proposing a linear model for depth estimation with non linear error. The model used is

$$
\begin{aligned}
& d=\hat{d}+\eta \\
& \hat{d}=\underline{W}^{T} \underline{f}
\end{aligned}
$$

where $\hat{d}$ denotes the predicted depth, $d$ is the ground truth depth, $\underline{W}^{T}$ denotes the weight estimated to compute the predicted depth and $f$ denotes the feature vector obtained. Here $\eta$ is the error of the model. Our task is to learn the weight vector $\underline{W}$ from the given data. The weight vector $\underline{W}$ is learned for all patches in a row taking information about the depth of same patches of row coming from different images. The weight vector $\underline{W}$ mentioned in the model is learned in an iterative process such that the error converges to zero. $(\eta \rightarrow 0)$

Let us assume that there are $l$ patches in a row and there are $n$ number of images in the data base. So we are getting $N=n l$ number of ground truth depth maps for learning $\underline{W}$. Using the data in the model we get :

$$
\begin{aligned}
& \underline{\hat{d}}=\underline{W}^{T} \mathbf{F}, \quad \text { where } \quad \underline{\hat{d}}=\left(\begin{array}{llll}
\hat{d}_{1} & \hat{d}_{2} & \hat{d}_{3} & \ldots \hat{d_{N}}
\end{array}\right)^{T} \\
& \text { and } \mathbf{F}=\left(\begin{array}{lllll}
\underline{f}_{1} & \underline{f}_{2} & \underline{f}_{3} & \cdots & \underline{f}_{N}
\end{array}\right)
\end{aligned}
$$

We start with an initial choice of $\underline{W}^{T}$ as $\underline{W}_{0}^{T}=\underline{d} \mathbf{F}^{-1}$, Where $\underline{d}$ is the ground truth depth vector form for all patches in a row and from all images. If $\mathbf{F}$ is not a square matrix than we can use generalized inverse of $\mathbf{F}$. 
Taking this initial choice of $\underline{W}_{0}^{T}$ and using features of each patch in a row of each image we estimate the depth by equation(2). Let it be $\hat{d}_{i}, \mathrm{i}=1,2 \ldots \mathrm{N}$. These estimates of depth are then subjected to correction to reduce the error. A non linear function, in particular, a $p^{\text {th }}$ degree polynomial, is fitted using the least square technique on the estimated depth $\hat{d}_{i}, i=1,2 \ldots N$ to get corrected estimate $\hat{\hat{d}}_{i}, i=1,2 \ldots N$. Hence, the new choice of $W^{T}$ is obtained as:

$$
\underline{W}_{n e w}^{T}=\underline{\hat{d}} F^{-1}
$$

where, $\quad \underline{\hat{\hat{d}}}=\left(\begin{array}{llll}\hat{\hat{d}}_{1} & \hat{\hat{d}}_{2} & \hat{\hat{d}}_{3} & \ldots \hat{\hat{d}}_{N}\end{array}\right)^{T}$ and $F$ is as defined earlier

This completes one iteration. In the next iteration, we will start with a choice of $\underline{W}^{T}$ as $\underline{W}_{n e w}^{T}$. We stop modification $\underline{W}^{T}$ if there is no significant.

The same learning procedure is adapted for predicting the absolute depth as well as the relative depth and hence the final depth is obtained. Here relative depth is the predicted difference of depths from the neighbors for a given patch.

\section{Implementation and Results}

The above mentioned methodology has been implemented on a large data set and the result obtained is comparable with the result presented in 11. A large data base of images along with their ground truth depth is available in [4]. For our experiments we have used 100 images of size 2272X1704 of outdoor scenes and there depth maps of size 51x78. 80 images from the above set were used for training and the rest 20 images were used for testing. The three monocular cues haze, texture edges and texture energy are extracted using the 17 Laws' masks [1] applied at three scales (one, one-third and one-ninth of the image). The feature vector for each patch is calculated for L1 and L2 norm using its 4 neighbors at 3 scales and the column feature vector for 4 patches. This results in a feature vector of size $646((((5 \times 3+4) \times 17=323) \times 2)=646)$.

The texture gradient filters used in Saxena et al. 1] are high pass filters oriented at $0,30,60,90,120$ and 150 degrees. In the new methodology, an Omni directional high pass filter is used for extracting texture gradient. Resulting in a reduction of the size of the image feature vector from 646 to $456((((5 \times 3+4)$ $\mathrm{x} 12=238) \mathrm{x} 2)=456$ ). The rest of the filters are same as used in Saxena et al. [1].

The resultant feature vector and the corresponding depth maps in the database were used to find the parameters of the model and the nonlinear function as described in Section 2. For the current implementation a polynomial of degree four is used which is obtained experimentally. So far we do not have any mathematical justification for the same. Note that the four iteration, as described in Section 2 appeared to be sufficient to estimate the model parameters.

The model of predicting relative depth is similar as of absolute depth. In absolute learning part learning was done by absolute value of depths for the patch and its neighboring patches. The difference of histograms of feature vectors are used as parameters to predict the difference of depth with neighbors of a 

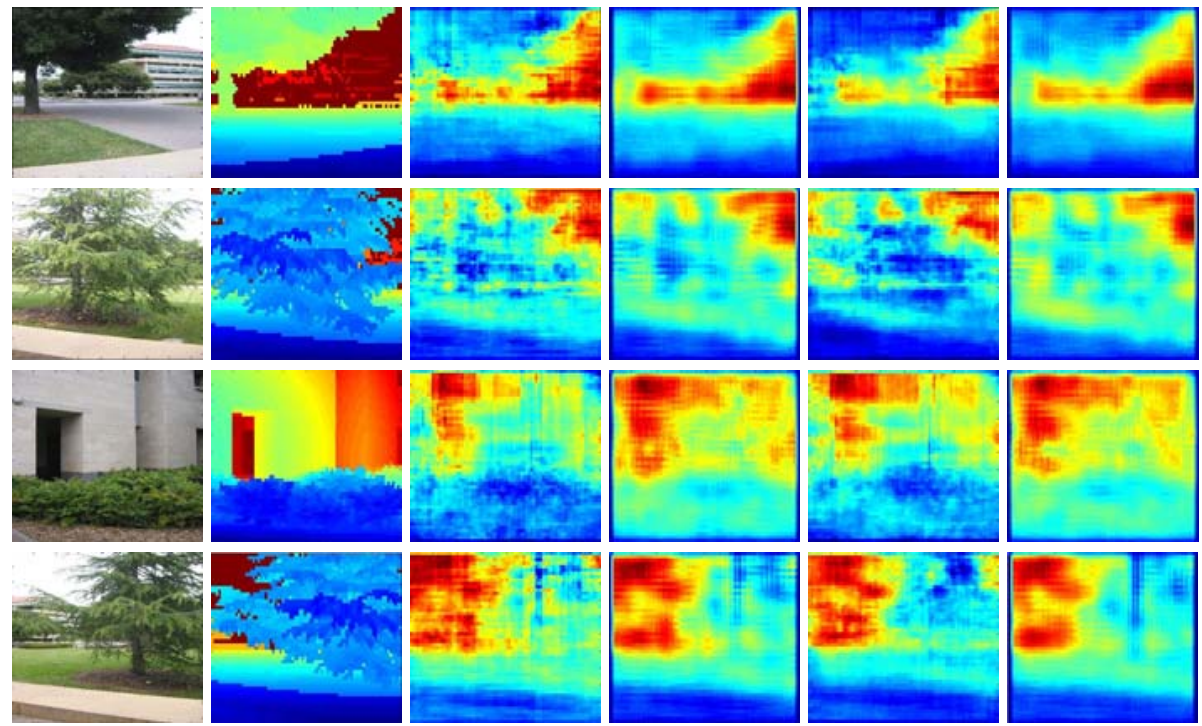

Fig. 3. A few test images along with their depth map predicted. Column 1 Depicts actual images. Column 2 Depicts actual ground truth depth maps. Column 3 Depicts absolute depth maps resulted from Saxena et al. 1]. Column 4 Depicts relative depth maps resulted from Saxena et al. 1]. Column 5 Depicts absolute depth maps predicted using new methodology. Column 6 Depicts relative depth maps predicted using new methodology.

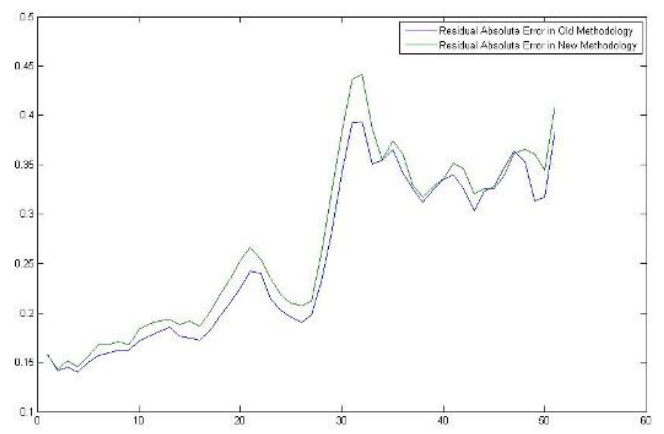

Fig. 4. Comparison of the absolute error in Saxena et al. [1] and after implementation of the new methodology.

given patch. Depth predicted for some of the test images are shown in Figure 3. Column 1 and 2 in Figure 3 shows the actual image and the ground truth depth maps respectively. The predicted depth maps generated by Saxena et al. [1] (column 3\& 4) and by the proposed methodology (column 5 \& 6) are also shown. Column 3 and 5 in Figure 3 corresponds to absolute depth maps and 
Column 4 and 6 in Figure 3 shows the depth maps after taking into account relative features.

The error comparison of the absolute depth prediction of Saxena et. al [1] and the absolute depth prediction after the implementation of the final filter is shown in the graph Figure 4. The change in edge detection filters reduces the sharp peaks in the relative difference prediction.

\section{Discussions and Conclusion}

Omni Directional Filters proposed here appear to be a promising filters. The result obtained is comparable with same presented in 1]. Moreover, the computational cost is reduced drastically by reducing the feature dimension. The 6 edge detection filters in [1] are replaced by a single Omni-directional high pass filter which reduce the size of feature vector, without significant change in the performance output. The time complexity of the model is evaluated to be $O(k . n)$, where $\mathrm{n}$ is the number of features in the feature vector and $\mathrm{k}$ is the number of iterations to adjust the parameters. The space complexity of the algorithm also reduces as the number of features in the feature vector decrease. The space complexity is evaluated to be $O(k . n)$, where $\mathrm{k}$ is the number of iterations performed to determine the parameters of the learning model and $n$ the number of features in the feature vector.

\section{References}

1. Saxena, A., Chung, S.H., Ng, A.Y.: Learning Depth from Single Monocular Images. In: NIPS (2005)

2. Saxena, A., Schulte, J., Ng, A.Y.: Learning Depth from Images using Monocular cues and stereo vision. In: ICJAI (2007)

3. Michels, J., Saxena, A., Ng, A.Y.: High speed obstacle avoidance using monocular vision and reinforcement learning. In: ICML (2005)

4. Saxena, A.: Stanford Range Image Data, http://ai.stanford.edu/ asaxena/learningdepth/data.html (last viewed on January 20, 2009)

5. Das, S., Ahuja, N.: Performance analysis of stereo, vergence, and focus as depth cues for active vision. IEEE Trans. Pattern Analysis \& Machine Intelligence 17, 1213-1219 (1995)

6. Lungeanu, D., Popa, C., Hotca, S., Macovievici, G.: Modeling biological depth perception in binocular vision: The local disparity estimation. Informatics for Health and Social Care 23(2), 131-143 (1998)

7. Van Amerongen, J., Ten Udink, C.A.J.: Model Reference Adaptive Autopilots for Ships. Automatica 11, 441-449

8. http://www.jhu.edu/wctb/coms/booklet/book5.htm (Visited on 19/4/2009 at 7:31 PM)

9. Scharstein, D., Szeliski, R.: A taxonomy and evaluation of dense two-frame stereo correspondence algorithms. Int'l. Journal of Computer Vision 47, 7-42 (2002)

10. Andreas, K., Mario, S., Konrad, K.: Segment-Based Stereo Matching Using Belief Propagation and a Self-Adapting Dissimilarity Measure. In: ICPR (2006) 